\title{
A novel NPR2 mutation (p.Arg388GIn) in a patient with acromesomelic dysplasia, type Maroteaux
}

\author{
Naoko Amano ${ }^{1,2}$, Hiroshi Kitoh ${ }^{3}$, Satoshi Narumi ${ }^{4}$, Gen Nishimura ${ }^{5}$, and Tomonobu Hasegawa ${ }^{1}$ \\ ${ }^{1}$ Department of Pediatrics, Keio University School of Medicine, Tokyo, Japan \\ ${ }^{2}$ Department of Pediatrics, Saitama City Hospital, Saitama, Japan \\ ${ }^{3}$ Department of Orthopedics Surgery, Aichi Children's Health and Medical Center, Obu, Japan \\ ${ }^{4}$ Department of Molecular Endocrinology, National Research Institute for Child Health and Development, Tokyo, \\ Japan \\ ${ }^{5}$ Center for Intractable Diseases, Saitama Medical University Hospital, Saitama, Japan
}

\begin{abstract}
Acromesomelic dysplasia, type Maroteaux (AMDM) is a congenital bone dysplasia characterized by disproportionate, acromesomelic shortening of the limbs and mild spondylar dysplasia. AMDM is caused by biallelic loss-of-function mutations in NPR2 encoding natriuretic peptide receptor-B. We report on a 25-yr-old Japanese woman with AMDM. Her height was $119.0 \mathrm{~cm}(-7.4 \mathrm{SD})$ and weight $35 \mathrm{~kg}(-2.3 \mathrm{SD})$. She had acromesomelic shortening of limbs and severe brachydactyly. Radiological examination showed that her metacarpals and phalanges were short and wide, and her vertebral bodies were mildly flattened. Molecular analysis revealed a novel homozygous NPR2 mutation (c.1163G>A, p.Arg388Gln). We performed in vitro functional studies using HA-tagged wild-type (WT) and Arg388Gln vectors (HA-WT-NPRB and HA-R388Q-NPRB). Cells expressing HA-R388Q-NPRB showed negligible cGMP responses to C-type natriuretic peptide (CNP) stimulation, indicating that the mutation led to severe loss-of-function. By immunofluorescence experiments under permeabilized conditions, HA-WT-NPRB was expressed on plasma membrane, while HA-R388Q-NPRB co-localized with an Endoplasmic Reticulum marker. Cells co-expressing R388Q and the WT exhibited lower responses under CNP treatment than cells co-expressing the WT and empty vectors. Thus, it was thought that R388Q caused a dominant-negative effect with a defect in cellular trafficking to the plasma membrane.
\end{abstract}

Key words: acromesomelic dysplasia, type Maroteaux, mutation, natriuretic peptide receptor-B, NPR2

\section{Introduction}

Acromesomelic dysplasia, type Maroteaux (AMDM, OMIM\#602875) is a rare congenital bone dysplasia characterized by disproportionate acromesimelic shortening of the limbs and mild spondylar dysplasia $(1,2)$. This disorder is caused by biallelic loss-of-function mutations in NPR2 encoding natriuretic peptide receptor-B (NPR-B) (3). Heterozygous loss-of-function mutations in NPR2 account for $0.4-6 \%$ of patients with idiopathic short stature (4-6). In contrast, gainof-function mutations in NPR2 cause an overgrowth syndrome termed epiphyseal chondrodysplasia, Miura type (\#615923) (7).

NPR-B is a single transmembrane receptor, consisting of the following domains; ligand binding domain, transmembrane domain, kinase homology domain, and guanylyl cyclase domain. The ligand of NPR-B, C-type natriuretic peptide (CNP), activates guanylyl cyclase, converting GTP into cGMP. This CNPNPR-B signaling plays a critical role in endochondral ossification, which is responsible for longitudinal growth in limbs and vertebrae $(8,9)$.

In this report, we present a patient with AMDM caused by a novel homozygous NPR2 mutation. Functional studies revealed that the mutant protein had a dominant-negative effect and defective cellular trafficking to the plasma membrane.

\section{Materials and Methods}

\section{Clinical report}

The proband was a 25-yr-old Japanese woman, who

Received: January 12, 2020 Accepted: March 6, 2020

Corresponding author: Naoko Amano, M.D., Ph.D., Department of Pediatrics, Keio University School of Medicine, 35 Shinanomachi, Shinjuku-ku, Tokyo 160-8582, Japan

E-mail: naokoam@z8.keio.jp 
A

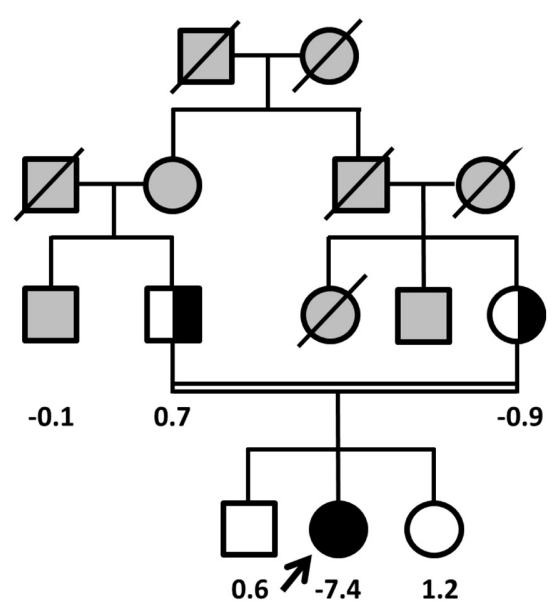

B

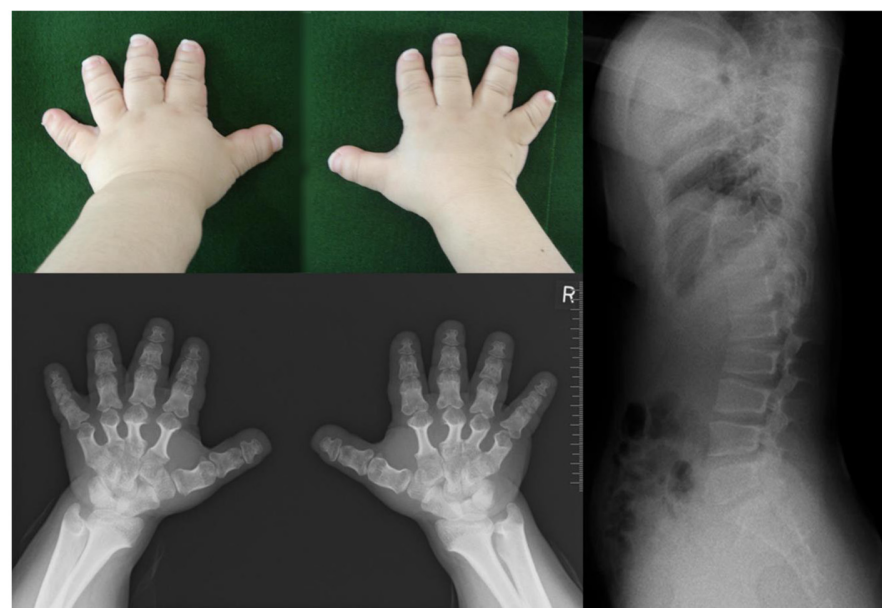

Fig. 1. A: Pedigree showing the family, including a patient with Acromesomelic dysplasia, type Maroteaux (AMDM). The values under the symbols show height SDS. NA denotes not available. B: A photograph and radiographs of the patient at $21 \mathrm{yr}$ of age. The photograph of her hands illustrates brachydactyly. The radiograph of her hands shows very short and wide metacarpals and phalanges. The radiograph of her lateral spine reveals platespondyly.

was born at term as the second child of a consanguineous couple of first-degree cousins (Fig. 1A). Her parents' heights were not short. The birth length of the proband was $46.6 \mathrm{~cm}$ (10th percentile) and birth weight was 3,570 $\mathrm{g}$ (90th-97th percentile). At $2 \mathrm{yr}$ of age, she was noted to have a short stature. At that point, the tentative clinical diagnosis was hypochondroplasia. At 21 yr of age, she was referred to us for a limb lengthening procedure. On physical examination, her height was $119.0 \mathrm{~cm}$ (-7.4 SD) and weight was $35 \mathrm{~kg}$ (-2.3 SD). She had acromesomelic limb shortening with severe brachydactyly (Fig. 1B). On radiological examination, her metacarpals and phalanges were very short and wide. Her vertebral bodies were mildly flattened, indicating platyspondyly (Fig. 1B). Based on these findings, she was clinically diagnosed with AMDM.

\section{Mutation detection}

We obtained written consent from the patient and her parents for molecular studies, which were approved by the Ethics Committee of Keio University School of Medicine. We extracted genomic DNA from the peripheral blood of the patient and her parents using standard techniques. All coding exons and flanking introns of NPR2 were analyzed by PCR-based DNA sequencing. The NPR2 genomic DNA sequence was based on NM_003995. Primer sequences and PCR conditions are available upon request.

\section{Functional characterization of a novel NPR2 mutation}

Plasmids, cell culture, and transfection: A hemagglutinin (HA)-tagged human wild-type (WT) NPRB expression vector (HA-WT-NPRB) has been described previously (10). A vector expressing mutant p.Arg388Gln (HA-R388Q-NPRB) was generated by site-directed mutagenesis (PrimeSTAR Mutagenesis Basal Kit, Takara, Tokyo, Japan). The mutant construct was verified by direct sequencing. COS7 cells were grown in DMEM supplemented with $10 \% \mathrm{FBS}$ and $1 \%$ penicillin/streptomycin at $37^{\circ} \mathrm{C}$ in $10 \% \mathrm{CO}_{2}$. Cells were subjected to transient transfection with Lipofectamine 2000 (Invitrogen ${ }^{\mathrm{TM}}$, Carlsbad, CA, USA) according to the manufacturer's protocol.

Measurements of CNP-dependent cGMP response: Cells were transiently transfected with each HA-NPRB vector. Twenty-four hours after transfection, the cells were incubated with or without $100 \mathrm{nM}$ of CNP-22 (Bachem, Ltd., Bubendorf, Switzerland) for $10 \mathrm{~min}$. The reaction was arrested with $200 \mu \mathrm{L}$ of $0.1 \mathrm{M} \mathrm{HCl}$. The cGMP levels of the lysate supernatant were measured using a competitive enzyme immunoassay (cyclic GMP Complete; Enzo Life Science, Farmingdale, NY, USA) according to the manufacturer's protocol.

Western blotting: Cells were transiently transfected with each HA-NPRB vector. Forty-eight hours after transfection, total cell lysates were subjected to $10 \%$ SDS-PAGE and transferred to PVDF membranes. HA-WT-NPRB and HA-R388Q-NPRB were detected by immunoblotting using a rat anti-HA antibody (clone 3F10; Roche Applied Science, Basel, Switzerland) and HRP-conjugated rabbit anti-rat IgG polyclonal antibody (Sigma-Aldrich, St. Louis, MO, USA). Immunoreactive bands were visualized using a chemiluminescence kit (ECL Western Blotting Substrate; Thermo Fisher Scientific, Waltham, MA, USA).

Subcellular localization analysis: Cells were transfected with either HA-WT-NPRB or HA-R388QNPRB construct with a green fluorescent protein-tagged endoplasmic reticulum (ER) marker construct. Forty- 


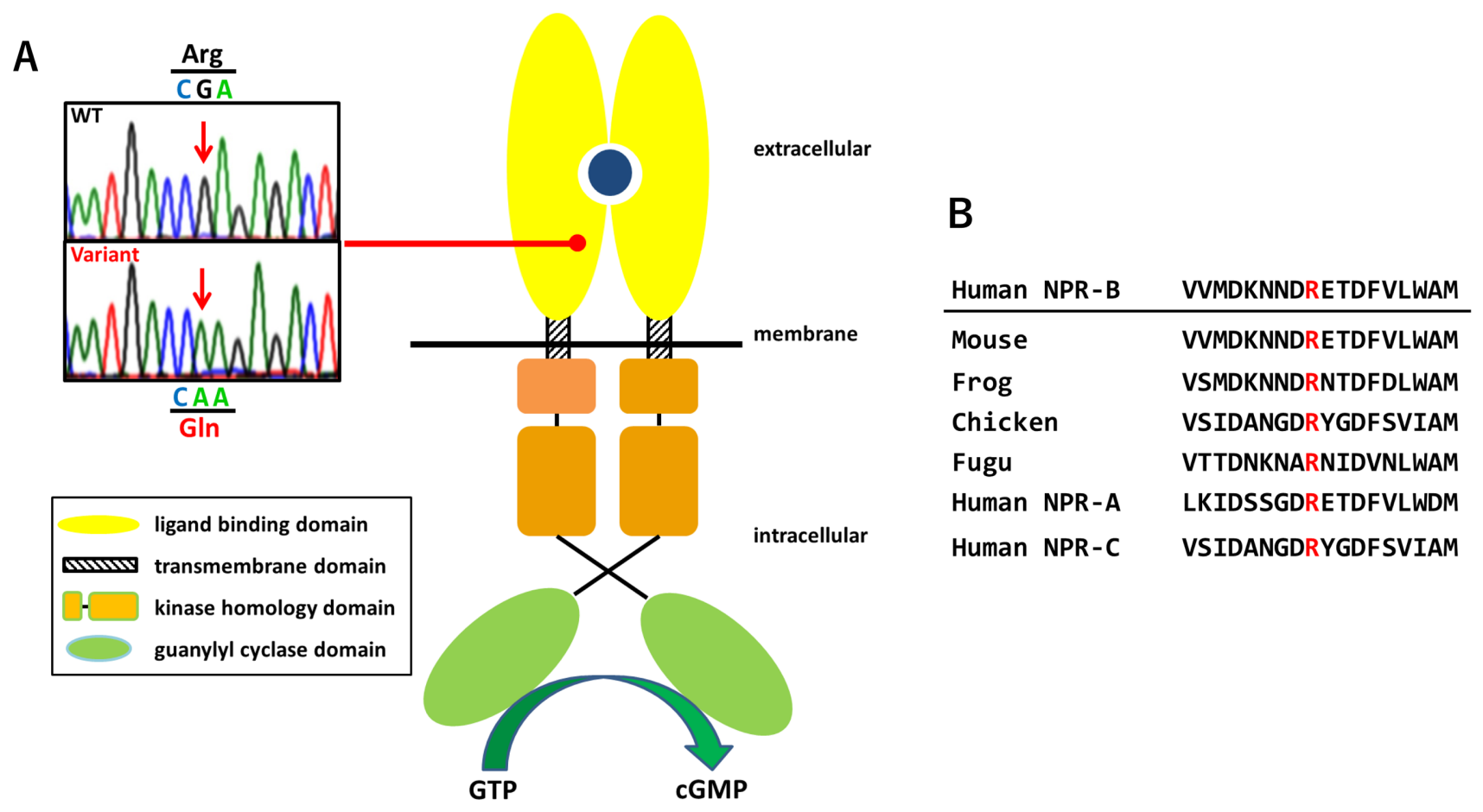

Fig. 2. Identification of a novel NPR2 mutation, R388Q. A: A schematic diagram of natriuretic peptide receptor-B(NPR-B) protein, which consists of an extracellular ligand binding domain, a transmembrane domain, a kinase homology domain, and a guanylyl cyclase domain. The blue closed circle shows C-type natriuretic peptide (CNP). The homozygous substitution of glutamine in place of Arg388 is indicated by an arrow on the chromatogram, and is located in the ligand binding domain. B: Sequence alignments surrounding the Arg388 amino acid residue are shown. Arg388 is conserved among NPR-B of terrestrial animals and other natriuretic peptide receptors (NPR-A and -C), which predominantly bind to atrial natriuretic peptide (ANP).

eight hours after transfection, the cells were fixed and treated with $0.1 \%$ Triton X-100 for permeabilization or left untreated. Fixed cells were incubated with a rat anti-HA antibody (3F10) at 1:100 dilution for 30 min., followed by a secondary antibody (Alexa Fluor 568 goat anti-rat IgG; Life Technologies, Carlsbad, CA, USA) at 1:100 dilution for $30 \mathrm{~min}$. Coverslips were mounted with VECTASHIELD Mounting Medium with DAPI (Vector Laboratory, Burlingame, CA, USA) and were observed under a TCS-SP5 confocal microscope (Leica, Microsystems, Mannheim, Germany).

\section{Results}

\section{Mutation detection}

We identified a novel homozygous missense NPR2 variant (c.1163G $>$ A, p.Arg388Gln) in the proband (Fig. 2A). The variant was not registered in the genome aggregation database (https://gnomad.broadinstitute. org/) or, dbSNP147 (http://www.ncbi.nlm.nih.gov/SNP/). Arg388, which is located in the ligand-binding domain, was highly conserved among vertebrate species and other natriuretic peptide receptors (NPR-A and -C) (Fig. 2B). These natriuretic receptors (NPR-A and -C) preferentially bind to atrial natriuretic peptide. The patient's parents were heterozygous for the mutation
p.R388Q (data not shown).

\section{Functional characteristics of R388Q mutation}

The CNP-dependent cGMP response of HA-R388QNPRB was as low as that of empty vector (Fig. 3A). To elucidate the mechanisms underlying the loss-offunction mutant (R388Q), we conducted further in vitro functional studies. Western blotting showed that the expression of R388Q was comparable to that of WT cells. However, R388Q showed only a lower-molecularweight band (lower band) compared with that of the WT, which showed two bands (Fig. 3B). Immunofluorescence experiments under non-permeabilized conditions revealed that the WT-NPRB protein was clearly expressed on the cell surface, while R388Q was not (Fig. 3C). Under permeabilized conditions, R388Q co-localized with an ER marker. Co-expression of R388Q and the WT protein led to a significant loss in CNP-dependent cGMP responses compared with that of the empty vector and WT, indicating a dominant-negative effect (Fig. 3D).

\section{Discussion}

We identified a novel NPR2 variant, c. $1163 \mathrm{G}>\mathrm{A}$, p.Arg388Gln in a patient with AMDM. She carried the variant in a homozygous state from her parents who were 
A

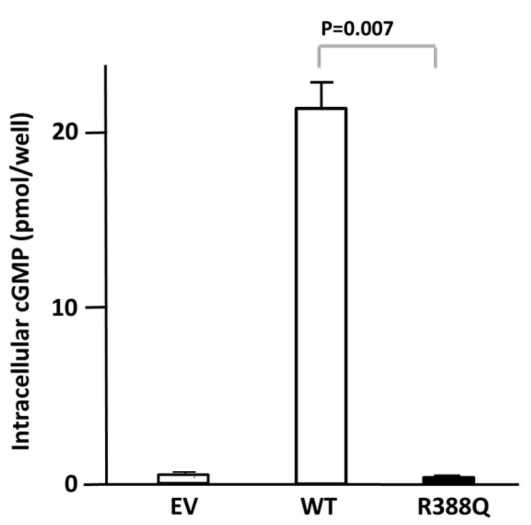

B

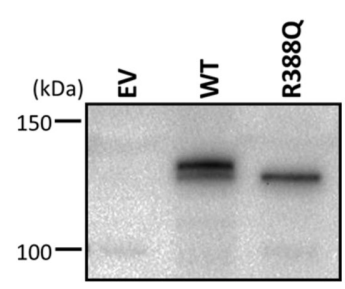

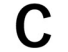
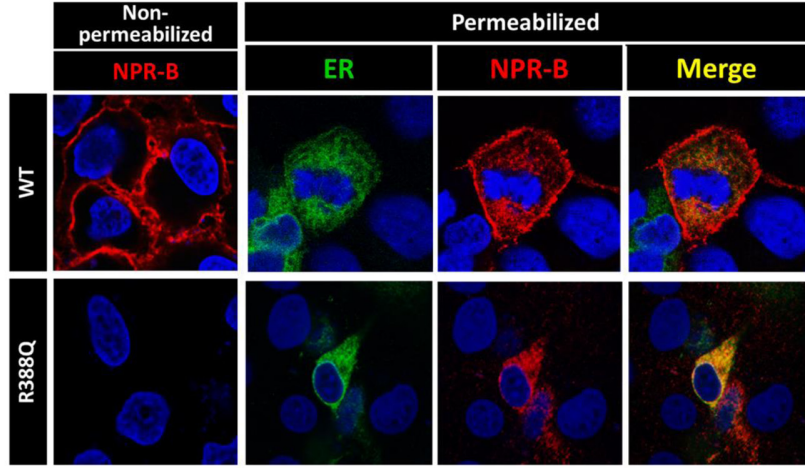

D

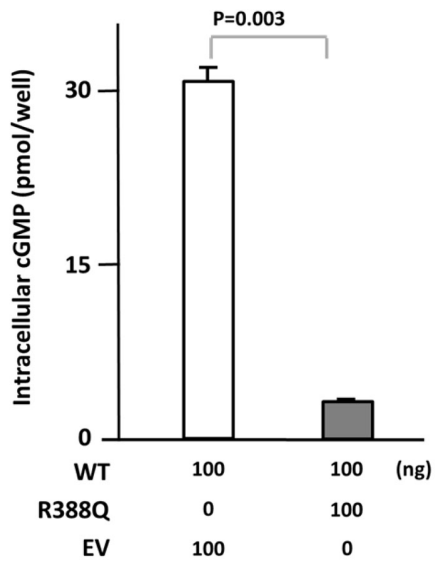

Fig. 3. Functional characterization of R388Q. A: C-type natriuretic peptide (CNP)-stimulated cGMP responses in cells transiently transfected with empty vector (EV), wild-type (WT) and R388Q mutant were evaluated. R388Q showed negligible response. Data are presented as the mean \pm SEM of triplicate samples, and are representative of three independent experiments. B: Immunoblotting analyses of HA-NPR-B proteins. The WT expressed two bands, whereas the R388Q mutant only presented the lower band. Migration of molecular mass markers (in $\mathrm{kDa}$ ) is shown on the left of each panel. C: Immunofluorescence analyses under non-permeabilized or permeabilized conditions. WT (red) showed clear plasma membrane expression in non-permeabilized cells, whereas R388Q (red) did not show any plasma membrane expression in non-permeabilized cells, but exhibited co-localized expression with the endoplasmic reticulum (green) marker in permeabilized cells. D: CNP-stimulated cGMP response in cells co-transfected with WT and R388Q.

first-degree cousins. We evaluated the cGMP response of p.R388Q to CNP treatment, which showed negligible cGMP production capacity. We confirmed that p.R388Q is a loss-of-function mutation.

The functional consequences of the disease-causing variant were ascertained by the following experiments. Immunofluorescence experiments showed that HA-WTNPRB was expressed on the plasma membrane, while HA-R388Q-NPRB colocalized with the ER marker, indicating defective intracellular trafficking from the ER to the plasma membrane. Moreover, immunoblotting analyses showed that HA-WT-NPRB expressed two immunoreactive bands, whereas HA-R388Q-NPRB expressed only a lower molecular weight band (lower band). This result raised the hypothesis that the mutant R388Q protein was not fully glycosylated in the Golgi apparatus. According to previous deglycosylation studies, the lower band was derived only from $\mathrm{N}$-glycosylated NPRB produced in the ER, while a higher molecular weight band (upper band) was derived from fully glycosylated NPRB produced in the Golgi apparatus $(5,11)$. It is assumed that full glycosylation in the Golgi apparatus creates correctly folded NPRB that is transported from the Golgi apparatus to the plasma membrane, while misfolded NPRB is retained in the ER. Such a hypothesis was also supported by the findings that HA-R388Q-NPRB colocalized with the ER marker. As previously reported, ER retention with defective trafficking to the plasma membrane is a common pathogenic consequence of NPRB mutants.

Moreover, the cGMP response of R388Q coexpressed with the WT protein showed that the mutant R388Q had a dominant-negative effect. Our previous co-expression studies involving HA-WT-NPRB demonstrated that the mutant R110C, which showed ER retention similarly to R388Q, interacted with WTNPRB in co-immunoprecipitation assays, and reduced the abundance of fully glycosylated NPRB as detected by immunoblotting assays. We speculated that the mutant R388Q exerted a dominant-negative effect by entrapping 
the WT receptor in the ER, similarly to R110C.

The heights of the patient's parents were not short. Recently, it has been reported that heterozygous NPR2 mutations are found in patients with short stature (4-6). Almost all of the mutations identified in a short-stature cohort that have been validated by functional studies exhibit a dominant negative effect $(4,5,13)$. Therefore, mutations with a dominant-negative effect would affect height, whereas it is unclear whether those without a dominant-negative effect (i.e. haploinsufficiency) could affect height. Moreover, heterozygous mutations with a dominant-negative effect have been observed in patients with disproportionate (i.e., mesomelic shortening of the arms), suggesting Léri-Weill dyschondrosteosis (13). Olney et al. studied height in a large family with AMDM. Heterozygous mutation carriers were on average 1.0 SD shorter than non-carrier family members. In this family, some carriers were taller than average, while others were less than $-2.0 \mathrm{SD}$ (12). Thus, the phenotypes of heterozygous carriers can be highly variable. This is because height is determined by polygenic and/ or multifactorial factors. NPR2 is a cardinal heightdetermining gene, but there are a number of other important genes, such as ACAN and SHOX (14).

\section{Conclusion}

In summary, we identified a novel loss-of-function mutation in a patient with AMDM. The identified mutation, R388Q, exerted a dominant-negative effect, with defective cellular trafficking to the plasma membrane.

Conflicts of Interest: Tomonobu Hasegawa received a research funding from Novo Nordisk and JCR Pharmaceuticals. Naoko Amano, Hiroshi Kitoh, Satoshi Narumi, Gen Nishimura have no conflict of interest.

\section{Acknowledgements}

We acknowledge Dr. Rumi Hachiya and Prof. Yoshihiro Ogawa for kindly providing HA-WT-NPR-B vector construct, and Prof. Takao Takahashi for fruitful discussions.

This work was partly supported by the Foundation for Growth Science, and by the Japan Agency for Medical Research and Development (AMED) (17bm0804012h0001)

\section{References}

1. Maroteaux P, Martinelli B, Campailla E. Acromesomelic dwarfism. Presse Med 1971;79: 1839-42 (In French). [Medline]

2. Langer LO, Garrett RT. Acromesomelic dysplasia. Radiology 1980;137: 349-55. [Medline] [CrossRef]

3. Bartels CF, Bükülmez H, Padayatti P, Rhee DK, van Ravenswaaij-Arts C, Pauli RM, et al. Mutations in the transmembrane natriuretic peptide receptor NPR-B impair skeletal growth and cause acromesomelic dysplasia, type Maroteaux. Am J Hum Genet 2004;75: 27-34. [Medline] [CrossRef]

4. Vasques GA, Amano N, Docko AJ, Funari MF, Quedas EP, Nishi MY, et al. Heterozygous mutations in natriuretic peptide receptor-B (NPR2) gene as a cause of short stature in patients initially classified as idiopathic short stature. J Clin Endocrinol Metab 2013;98: E1636-44. [Medline] [CrossRef]

5. Amano N, Mukai T, Ito Y, Narumi S, Tanaka T, Yokoya S, et al. Identification and functional characterization of two novel NPR2 mutations in Japanese patients with short stature. J Clin Endocrinol Metab 2014;99: E713-8. [Medline] [CrossRef]

6. Wang SR, Jacobsen CM, Carmichael H, Edmund AB, Robinson JW, Olney RC, et al. Heterozygous mutations in natriuretic peptide receptor-B (NPR2) gene as a cause of short stature. Hum Mutat 2015;36: 474-81. [Medline] [CrossRef]

7. Miura K, Namba N, Fujiwara M, Ohata Y, Ishida H, Kitaoka T, et al. An overgrowth disorder associated with excessive production of cGMP due to a gain-of-function mutation of the natriuretic peptide receptor 2 gene. PLoS One 2012;7: e42180. [Medline] [CrossRef]

8. Yasoda A, Ogawa Y, Suda M, Tamura N, Mori K, Sakuma Y, et al. Natriuretic peptide regulation of endochondral ossification. Evidence for possible roles of the C-type natriuretic peptide/guanylyl cyclase-B pathway. J Biol Chem 1998;273: 11695-700. [Medline] [CrossRef]

9. Schulz S. C-type natriuretic peptide and guanylyl cyclase B receptor. Peptides 2005;26: 1024-34. [Medline] [CrossRef]

10. Hachiya R, Ohashi Y, Kamei Y, Suganami T, Mochizuki H, Mitsui N, et al. Intact kinase homology domain of natriuretic peptide receptor-B is essential for skeletal development. J Clin Endocrinol Metab 2007;92: 4009-14. [Medline] [CrossRef]

11. Wang W, Song MH, Miura K, Fujiwara M, Nawa N, Ohata Y, et al. Acromesomelic dysplasia, type maroteaux caused by novel loss-of-function mutations of the NPR2 gene: Three case reports. Am J Med Genet A2016;170A: 426-34. [Medline] [CrossRef]

12. Olney RC, Bükülmez H, Bartels CF, Prickett TC, Espiner EA, Potter LR, et al. Heterozygous mutations in natriuretic peptide receptor-B (NPR2) are associated with short stature. J Clin Endocrinol Metab 2006;91: 1229-32. [Medline] [CrossRef]

13. Hisado-Oliva A, Garre-Vázquez AI, Santaolalla-Caballero F, Belinchón A, Barreda-Bonis AC, Vasques GA, et al. Heterozygous NPR2 mutations cause disproportionate short stature, similar to Léri-Weill dyschondrosteosis. J Clin Endocrinol Metab 2015;100: E1133-42. [Medline] [CrossRef]

14. Andrade AC, Jee YH, Nilsson O. New genetic diagnoses of short stature provide insights into local regulation of childhood growth. Horm Res Paediatr 2017;88: 22-37. [Medline] [CrossRef] 